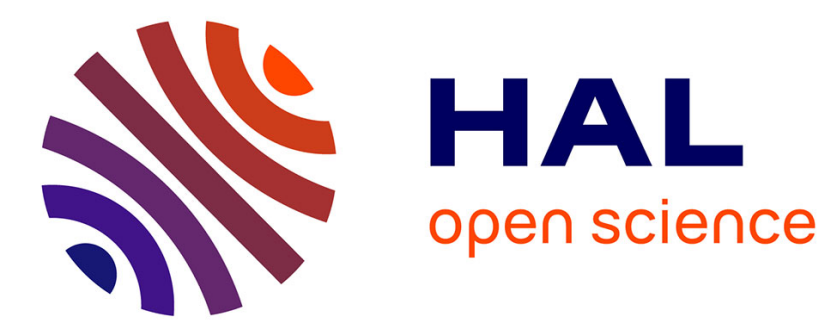

\title{
Les mashups: une illustration de l'agilité en marketing
} Maria Mercanti-Guérin

\section{To cite this version:}

Maria Mercanti-Guérin. Les mashups : une illustration de l'agilité en marketing. Décisions Marketing, 2013, 71, pp.125-135. 10.7193/DM.071.125.135 . hal-02054307

\section{HAL Id: hal-02054307 https://hal.science/hal-02054307}

Submitted on 4 Mar 2019

HAL is a multi-disciplinary open access archive for the deposit and dissemination of scientific research documents, whether they are published or not. The documents may come from teaching and research institutions in France or abroad, or from public or private research centers.
L'archive ouverte pluridisciplinaire HAL, est destinée au dépôt et à la diffusion de documents scientifiques de niveau recherche, publiés ou non, émanant des établissements d'enseignement et de recherche français ou étrangers, des laboratoires publics ou privés. 


\section{Les mashups : une illustration de l'agilité en marketing}

\section{Maria Mercanti-Guérin}

Maître de conférences

Adresse professionnelle

Conservatoire national des arts et métiers

2 rue Conté

75002 Paris

\section{RUBRIQUE : DIGITAL}

L'auteur remercie la société Artik Consulting pour le support technique qu'elle a bien voulu lui accorder dans le cadre de la rédaction de cet article. Elle remercie également le Professeur Christophe Benavent pour ses conseils et suggestions d'amélioration. 


\title{
Les mashups : une illustration de l'agilité en marketing
}

Résumé : le mashup est une application composite essentiellement connue pour ses utilisations en géomarketing. Pourtant, les mashups se généralisent au sein des sites de ecommerce et apportent des services qui vont bien au-delà de la cartographie. L'objectif de cet article est d'illustrer l'apport de ces nouvelles applications sur l'agilité en marketing. Les fonctionnalités des sites mashups et les développements futurs possibles sur le consommateur sont abordés. Les mashups offrent une gamme de services professionnels allant du CRM au management des campagnes publicitaires et ont un impact positif sur le travail collaboratif et l'agilité organisationnelle.

Mots-clés : mashups, wikis, services, e-commerce, agilité

\begin{abstract}
Mashup is a Web application primarily known for its use in cartographic data. However, mashups became widespread among the eRetail and provide services that go well beyond mapping. The objective of this research is to understand and measure the implications of these new applications on marketing agility. The features of the mashup sites and possible future developments for consumers are drawn. Mashups offer a full range of professional services from CRM to advertising management and positively impact collaborative work and business agility.
\end{abstract}

Keywords: mashups, wikis, services, eRetail, agility 


\section{Les mashups : une illustration de l'agilité en marketing}

Les mashups sont des applications composites qui suscitent un intérêt croissant parmi les praticiens et la recherche académique (5). Les mashups ont été développés initialement par les consommateurs eux-mêmes. Ils correspondent à une philosophie de l'open-source au sein de laquelle le consommateur co-crée les services qu'il désire. Cette notion de bricolage rendue possible par la facilité d'utilisation des outils du Web 2 .0 est étudiée par de nombreux auteurs dans le domaine de la distribution $(8,3)$. Un mashup permet d'associer des informations et/ou des contenus issus de différentes sources (sites Web par exemple) pour apporter une information nouvelle à l'utilisateur. Ainsi, Amazon ou eBay ont conçu leur suivi de commande autour d'un mashup permettant de mixer les informations du transporteur et celles de l'entreprise. Plus de six mille mashups sont, aujourd'hui, répertoriés. Ces derniers sont utilisés en géomarketing, dans l'industrie de la recommandation ou en logistique. Leur large champ d'application a une influence directe sur bon nombre de services proposés au consommateur. L'institut Forrester (13) évalue ce marché à 700 millions de dollars à 1'horizon 2013. Plusieurs facteurs peuvent expliquer cet engouement. Outre une meilleure gestion de la sécurisation des données grâce au lancement sur le marché de produits comme celui d'IBM, les entreprises trouvent dans les mashups des sources de performance, d'économie et, surtout, d'agilité importantes. En effet, la combinaison d'applications composites internes avec des données externes permet d'améliorer considérablement un certain nombre de processus allant du suivi du client à la logistique. L'objectif de cet article est de présenter les nombreuses possibilités qu'offrent les mashups en matière de CRM, de création de communautés ou de présentation produit sur les catalogues des sites marchands. Plus généralement, ces nouveaux services semblent être un remède à la lourdeur des processus et des outils informatiques traitant de gestion de la relation client ou utilisés dans l'élaboration des solutions de e-commerce. Les mashups redonneraient aux entreprises une nouvelle agilité leur permettant de répondre au mieux aux besoins des consommateurs. Dans une première partie, nous définirons ce qu'est un mashup, ses origines, son mode de construction. Dans une deuxième partie, nous détaillerons les liens conceptuels existant entre agilité et systèmes d'information et plus précisément agilité et mashups. Dans une troisième partie, nous donnerons des exemples des différents services mashups et des réponses qu'ils peuvent apporter aux besoins d'agilité des entreprises. Nous conclurons par une réflexion sur 
l'avenir des mashups, leur capacité à enrichir le concept d'agilité mais aussi les risques inhérents à leur utilisation.

\section{Les mashups : définition et origine}

\section{Qu'est-ce qu'un mashup?}

Pour l'opérateur de téléphonie Orange ${ }^{1}$ « un mashup désigne un service qui combine des fonctionnalités ou du contenu en provenance de différents sites. Cette application permet ainsi de créer un service original grâce à l'apport des fonctionnalités et des contenus de fournisseurs tiers ». Le nouveau service (mashup) ainsi créé peut être à son tour enrichi de contenus générés par les utilisateurs et amélioré avec l'ajout de nouvelles fonctionnalités proposées par d'autres développeurs ». Les mashups peuvent être :

Des mashups de présentation qui agrègent des données de plusieurs sources et permettent à l'utilisateur de consulter sur la même page différentes informations sans lien entre elles. Le portail Netvibes qui intègre les messages des différentes boîtes mail de l'utilisateur, la presse en ligne, les offres promotionnelles des marques que le consommateur souhaite suivre, les widgets $^{2}$ météo ou cours de bourse en est une illustration.

Des mashups de données qui permettent une interaction entre les différentes données issues de sources diverses. Ces mashups sont très souvent utilisés dans la construction de tableaux de bord personnalisables en fonction des profils métier des utilisateurs. La combinaison de ces données permet de créer de nouveaux services. IBM Lotus Mashups permet aux utilisateurs professionnels d'assembler du contenu personnel, métier et Web. Les mashups de données sont des systèmes ouverts qui donnent la possibilité de rechercher et intégrer des informations externes, notamment celles issues du Web 2.0.

La synthèse de ces deux types ${ }^{3}$ de mashups (données et présentation) permet des applications variées en matière, notamment, de recommandation, de géolocalisation et de personnalisation :

\footnotetext{
${ }^{1}$ Cette définition est tirée du Wiki développé par Orange pour vulgariser le concept de mashups et les mashups mobile proposés par Orange : http://api.orange.com/wiki/index.php/Qu'est-ce_qu'un_mashup

${ }^{2}$ Les widgets sont les mashups les plus connus par le grand public. Contraction de windows et gadget, ces petits programmes donnent la météo ou les cours de bourse. Ils sont facilement exportables sur un site ou sur le bureau de son ordinateur personnel.

${ }^{3}$ Un troisième type de mashup est souvent cité par les praticiens à savoir les business mashups. Ces mashups permettent à l'ensemble des acteurs métier d'intervenir sur les processus, d'enrichir les données, de modifier les présentations en vue d'obtenir une meilleure ergonomie. Ils se rapprochent, à notre sens, plus des services Web que des mashups.
} 
- La recommandation donne la possibilité de gérer les communautés en développant la notion de proximité entre membres. Ainsi, les informations sur les contributeurs (photos, vidéos, localisation) rendent extrêmement actifs les estimations collaboratives et renforcent le sens communautaire.

- La géolocalisation est couplée à la photo, la vidéo. Par ailleurs, elle peut être source de vraies économies financières pour les internautes en permettant la recherche de magasins de proximité (affranchissement d'un long trajet ou de frais de livraisons)

- La personnalisation des requêtes, de la recherche d'information, des portails collaboratifs modifie la navigation et réduit le temps consacré à la recherche. Ce n'est plus l'internaute qui va à la recherche de l'information mais l'inverse. Celle-ci est, en outre, actualisée.

\section{Comment naît un mashup?}

Un mashup est composé d'API (Application Program Interface) qui sont généralement au cœur de la construction du mashup. Les API sont des interfaces permettant d'appeler une application à partir d'une autre (hybridation). Les mashups sont donc issus des multiples possibilités de couplage d'API, certaines très connus (les API de Google allant de la géolocalisation en passant par la traduction), d'autres plus confidentielles. Le site Programmable $W e b$, site de référence des mashups visualise une matrice intégrant l'ensemble des mashups existant et les API dont ils sont issus. Cette matrice donne une idée de la diversité des combinaisons possibles. De nombreux éditeurs de contenu comme Google, Yahoo, Amazon ou eBay fournissent librement leurs API, leur intérêt étant de devenir incontournables sur le marché (encadré 1).

\section{Encadré 1 : Les API favoris dans la création des mashups}

Les sociétés de vente en ligne les plus connues ont fait une utilisation très en amont de la technologie mashup. Ainsi, l'API d'eBay permet d'intégrer des fonctionnalités telles que l'analyse et le suivi des ventes, la création de catalogues. L'API d'Amazon rend possible le ciblage comportemental. L'API de FedEx permet à un commerçant de présenter sur son propre site Web l'état d'avancement d'une livraison. Moins connu mais tout aussi représentatif de l'esprit mashup, le site Earlymiser regroupe les moteurs de recherche d'Amazon, d'eBay, de Yahoo Shopping et de Shopping.com. 
Certains mashups peuvent se constituer autour d'un nombre important d'API et ce, en fonction du service qu'ils souhaitent rendre à leur utilisateur. Research and Compare qui est un site mashup permettant de comparer des millions de produits entre eux de différentes marques et de différents magasins tout en prenant en compte l'avis du réseau social du consommateur s'est construit à partir de plusieurs API (tableau 1) :

\section{Tableau 1 : illustration des types d'API mobilisés dans la construction d'un site mashup}

\begin{tabular}{|c|c|}
\hline API UTILISEES & AVANTAGES MARKETING \\
\hline $\begin{array}{l}\text { Api de Facebook } \\
\text { Permet de rajouter les photos de profil des utilisateurs, les achats effectués } \\
\text { par les amis - permet d'intégrer Facebook Connect à la page d'accueil ainsi } \\
\text { que la possibilité de suivre la page Fans de l'application }\end{array}$ & $\begin{array}{l}\text { Constitution d'une base de données qualifiée } \\
\text { de clients et prospects } \\
\text { Amélioration de l'activité communautaire } \\
\text { de son site } \\
\text { Augmentation de la viralité de son contenu }\end{array}$ \\
\hline $\begin{array}{l}\text { Api de Twitter } \\
\text { Permet d'intégrer l'icône de suivi du compte Twitter à la page d'accueil ou } \\
\text { de récupérer les tweets parlant du contenu mis en ligne }{ }^{4}\end{array}$ & Enrichissement de l'interactivité de son site \\
\hline $\begin{array}{l}\text { API de Social Oomph } \\
\text { Permet de gérer le compte Twitter de l'application : messages automatisés } \\
\text { de remerciement, abonnements, mise en évidence des fils Twitter ayant le } \\
\text { plus de suiveurs }\end{array}$ & $\begin{array}{l}\text { Automatisation de sa stratégie sociale } \\
\text { Identification des influenceurs }\end{array}$ \\
\hline $\begin{array}{l}\text { API de You Tube } \\
\text { Permet d'accéder au moteur de recherche des vidéos présentes sur le site } \\
\text { par catégorie et par mot-clé }\end{array}$ & $\begin{array}{l}\text { Amélioration de l'ergonomie du site et de } \\
\text { l'expérience utilisateur }\end{array}$ \\
\hline $\begin{array}{l}\text { API de Mail Chimp } \\
\text { Permet de gérer la relation client : mails de confirmation, formulaires } \\
\text { d'abonnement etc... }\end{array}$ & $\begin{array}{l}\text { Mise en place d'un marketing du } \\
\text { déclenchement (trigger marketing) }\end{array}$ \\
\hline
\end{tabular}

Les possibilités des API sont représentatives des réponses agiles que les technologies offrent au marché. Elles sont décuplées par l'utilisation des mashups qui représentent des voies innovantes de développement d'une nouvelle agilité.

\footnotetext{
${ }^{4}$ TF1 via son site Web mytf1.fr a inauguré en 2011 un concept entièrement nouveau : le tweet replay. II permet, pour chaque diffusion, de consulter les tweets des internautes ayant visionné le programme et de suivre en différé leurs commentaires et réactions. Cette télévision sociale permettrait une augmentation de l'audience de $11 \%$ en moyenne selon une étude du cabinet américain NM Incite.
} 


\section{Les mashups et le concept d'agilité : la réponse du marché aux attentes des consommateurs}

\section{Agilité des systèmes d'information et marketing}

La lourdeur de bon nombre de processus liant systèmes d'information et marketing est pointée du doigt par de nombreux chercheurs (11). Les systèmes d'information donnent au marketing une meilleure appréhension du client (gestion de la relation client, évaluation de la publicité et de la promotion, rentabilité des produits etc...). Néanmoins, les systèmes d'information marketing rencontrent des difficultés dans leur nécessaire adaptation à un environnement en perpétuelles mutations. Les exemples sont nombreux de cette lenteur d'adaptation. Ainsi, l'arrivée du mobile implique une mutation très rapide des sites marchands vers des sites mobile puis des applications, mutation que les acteurs du marché peinent à réalisếr Ces retards ont pour résultat un fort développement de l'open-source et du bricolage réalisé par le consommateur lui-même d'outils lui apportant le service recherché. L'agilité définie comme une réponse délibérée des entreprises leur permettant « de prospérer dans un environnement concurrentiel dont les opportunités de marché changent continuellement et de manière imprévisible» $(2,6)$ est redécouverte par bon nombre de sociétés dont celles issues des nouvelles technologies. L'agilité organisationnelle permet de mettre en œuvre des ajustements constants et rapides imposé par un environnement en perpétuelle mutation. L'attitude des entreprises agiles est proactive et s'appuie sur l'innovation. Trois caractéristiques de l'agilité organisationnelle sont à souligner :

- La capacité à mobiliser une réponse rapide aux attentes des consommateurs et notamment une flexibilité réactive comme la possibilité de mettre sur le marché des produits et des services conçus comme des combinaisons individualisées d'offres

- L'aptitude à «lire» le marché en mettant en place des systèmes de veille au sein desquels les informations disponibles sont transformées en projets créateurs de valeur

- L'intégration de l'apprentissage organisationnel aux problématiques de l'agilité en développant des compétences spécifiques ou en facilitant le transfert de connaissances au sein des équipes voire même au sein des communautés de clients

\footnotetext{
${ }^{5}$ L'émergence du multi-support et la difficulté à adapter automatiquement un site aux différentes tailles d'écran poussent de nombreuses entreprises à adopter des méthodes agiles permettant d'homogénéiser leur système d'information. C'est le cas de Cadremploi ou du Figaro.
} 
Flexibilité réactive, aptitude à lire le marché et apprentissage organisationnel sont repris dans les nombreuses utilisations des mashups par les entreprises à savoir :

- L' e-commerce avec, notamment, une meilleure expérience utilisateur et une mise sur le marché des produits la plus performante possible

- La connaissance et l'analyse du client et son corollaire, l'amélioration de son CRM

Ces axes s'insèrent dans les deux principales directions de recherche ayant trait au concept d'agilité. Elles ont été conceptualisées et vérifiées empiriquement par les recherches les plus récentes portant sur l'agilité (9). Conscients du caractère dispersé des résultats traitant des manifestations de l'agilité en entreprise, les chercheurs s'orientent vers des modélisations du concept d'agilité selon deux dimensions: la capacité à sentir et comprendre les besoins du consommateur notamment à travers les logiques de CRM et la capacité à répondre à ses besoins notamment à travers la mise sur le marché des produits.

\section{L’agilité-mashup : des combinaisons individualisées d'offres de services}

\section{Les mashups du CRM ou la capacité à connaître et analyser les besoins du consommateur}

Cette prise en compte des besoins des consommateurs peut se faire selon deux directions :

Une vision centrée sur le client et, notamment, sur ses activités en dehors de sa relation à l'entreprise: participation à des forums, expression de son mécontentement ou de sa satisfaction à l'égard de la marque sur les réseaux sociaux etc...

Une vision centrée sur la concurrence et, notamment, sur les réactions de ses clients à ses actions promotionnelles et à celles de ses concurrents

\section{- Le CRM-mashup : une lecture de son marché et de celui de ses concurrents}

Des acteurs majeurs de la relation client comme Sales Force ont fait des mashups la pierre angulaire de leurs outils de CRM. Les nouveaux outils mashups de gestion de la relation client permettent de :

Coordonner les équipes concernées par le CRM (commercial, marketing, comptabilité) grâce à une seule interface-client regroupant identité, localisation, commandes, facturations, 
incidents-clients, relations avec le service après-vente. Cette coordination permet d'éviter la duplication des comptes-clients et de réagir plus rapidement en cas de problème.

Réduire les coûts d'intégration. Les sociétés comme Convertigo, spécialisées dans la commercialisation de mashups insistent sur le caractère «non-expert» des mashups, simples à concevoir, à implémenter et à faire évoluer.

Géolocaliser ses clients, examiner de façon contextuelle leur consommation, attirer de nouveaux clients sur certaines zones pré-définies. La personnalisation géographique de la recherche (le produit recherché par le consommateur est indiqué sur Internet dans sa zone de chalandise avec l'adresse exacte du magasin le plus proche) est rendue possible par l'utilisation de l'API fournie par Google Map.

Donner aux actions promotionnelles un caractère plus immédiat grâce à l'utilisation de widgets promotionnels sur sa base de données-clients.

Personnaliser les services rendus aux clients en les adaptant à leur comportement de consommation. Ainsi, Voice Mashup d'Orange permet la création d'annonces vocales en fonction de sa disponibilité mais aussi de ses différents canaux de communication (portable, réseaux sociaux etc.).

Les données du carnet d'adresse des commerciaux itinérants sont combinées avec une application de gestion des ventes permettant de connaitre l'état des commandes clients, le montant de leurs impayés, leur niveau de satisfaction. L'appareil photo couplé à une application de gestion des livraisons donne la possibilité aux transporteurs de documenter le bon état des colis livrés. Ces «combinatoires » de données améliorent la vision marché de l'entreprise. Le tableau suivant illustre quelques extensions de service que les mashups peuvent offrir au CRM (tableau 2). Le CRM enrichi de différents mashups (sociaux, temporels, géographique ...) permet de créer des applications multi-sources, facilement personnalisables par les différents acteurs (commerciaux, services marketing, personnels des centres d'appel), partageables et adaptables. Il renforce le caractère opérationnel des outils CRM en donnant une vision du client unique, actualisée, une vue à $360^{\circ}$ intégrant les applications du Web 2.0. Ainsi, un environnement se crée, plus collaboratif et ce, notamment grâce au CRM social. Les présentations, le travail d'équipe, les bilans de campagne peuvent être intégrés à des portails et implémentés sur des postes de travail extrêmement différents de l'utilisateur-métier en passant par la direction générale. La création d'un CRM-mashup 
permet d'assembler à coûts extrêmement économiques différentes applications du système d'information interne de l'entreprise à d'autres applications externes.

\section{Tableau 2 : illustrations des extensions de service possibles grâce aux mashups}

Source: adapté de «Le mashup d'entreprise booster de CRM», Convertigo, Livre blanc, février 2010

\begin{tabular}{|c|c|c|}
\hline & $\begin{array}{l}\text { Fonctionnalités classiques d'un } \\
\text { CRM }\end{array}$ & $\begin{array}{l}\text { Exemple de fonctionnalités améliorées grâce aux } \\
\text { mashups }\end{array}$ \\
\hline $\begin{array}{l}\text { Gestion des } \\
\text { références } \\
\text { clients }\end{array}$ & $\begin{array}{l}\text { Gestion des comptes, des contacts } \\
\text { Gestion de l'activité des ventes des } \\
\text { partenaires, des territoires } \\
\text { Prévision des ventes, analyse de la } \\
\text { concurrence }\end{array}$ & $\begin{array}{l}\text { Ajout de la photo du prospect et de ses informations } \\
\text { personnelles captées sur les réseaux sociaux } \\
\text { Téléchargement de widgets affichant la météo locale, la } \\
\text { circulation routière jusqu'au site du client } \\
\text { Mise en place de mashups d'alerte à l'aide d'un flux } \\
\text { RSS, observation sur les alertes Google des informations } \\
\text { financières et stratégiques des concurrents }\end{array}$ \\
\hline $\begin{array}{l}\text { Service } \\
\text { clients }\end{array}$ & $\begin{array}{l}\text { Centres de service clients, gestion des } \\
\text { contacts clients ; réponses aux emails, } \\
\text { gestion des appels }\end{array}$ & $\begin{array}{l}\text { Visualisation des stocks disponibles avec des scripts de } \\
\text { vente et des offres promotionnelles pour que les } \\
\text { commerciaux puissent proposer des produits livrables } \\
\text { dans les délais imposés par le client ou mettre en avant } \\
\text { des produits à forte rentabilité } \\
\text { Gestion des incidents clients grâce à des mashups } \\
\text { «temporels » permettant de visualiser l'historique client, } \\
\text { les actions correctives mises en place, la disponibilité du } \\
\text { personnel de maintenance }\end{array}$ \\
\hline $\begin{array}{l}\text { Opérations } \\
\text { marketing }\end{array}$ & $\begin{array}{l}\text { Organisation de salons et invitation } \\
\text { de ses prospects et clients }\end{array}$ & $\begin{array}{l}\text { Combinaison d'informations sur le lieu d'évènements } \\
\text { (hôtels, centres de conférence) avec les informations } \\
\text { logistiques et budgétaires }\end{array}$ \\
\hline
\end{tabular}

\section{La mesure des performances des campagnes}

La mesure des performances des campagnes s'appuie, de plus en plus, sur l'utilisation des mashups. Les mashups les plus connus de gestion de campagne sont ceux élaborés à partir des API de Yahoo ou de Google utilisant des éditeurs de mashups comme Adwords Editor de Yahoo ou Google Mashup Editor. Ces éditeurs permettent de créer à travers des langages accessibles comme le langage HTML des mashups qui, notamment, enrichissent les suivis et 
les bilans de campagne. Dès lors, il est possible de mesurer la performance de son coût au clic ou de chaque mot clé acheté en fonction de ses différents segments, de la localisation géographique des acheteurs, de leur niveau de satisfaction captée sur les forums, des évolutions de prix des concurrents etc...

\section{Les mashups de l'e-commerce ou la capacité à répondre aux besoins des consommateurs}

Les mashups de l'e-commerce vont de l'agrégation d'information au zooming. Ils sont, pour la majorité, conçus dans une optique de facilitation de l'expérience utilisateur. Cette expérience utilisateur est enrichie par des combinaisons multiples de e-services. Ainsi, chez Aislefinder.com, le consommateur envoie sa liste de courses, à charge pour le site de localiser les magasins qui disposent du produit ou offrent des réductions de prix. Les mashups révolutionnent les achats en ligne en assistant le consommateur dans sa navigation. Cette révolution trouve son aboutissement dans une gestion typiquement « mashup » de catalogues aux services et fonctionnalités de plus en plus étendues. Nous citerons, notamment, la présentation des produits au sein des catalogues virtuels, le zooming et la gestion du multisupport

\section{- La présentation des produits au sein des catalogues virtuels}

Le sourcing de catalogues ou « data harvesting » a pour objectif d'intégrer les données produits des sites du fournisseur directement sur le site du marchand. Des sociétés comme Convertigo proposent des solutions permettant de combiner différents services comme l'actualisation automatique des prix des produits ou leur disponibilité. Les comparateurs de prix comme TooCamp utilisent les mashups pour enrichir les informations produits en temps réel. Des acteurs plus spécialisés comme les bibliothèques recourent à des mashups proposés

par des plates-formes comme Iguana. Conçue comme une plate-forme de mashups, Iguana permet de traiter le site Web de la bibliothèque et son catalogue comme deux entités fusionnées au sein d'un même environnement. Les usagers peuvent personnaliser leur navigation et décider des contenus qu'ils souhaitent intégrer dans des sites comme IGoogle grâce à un mashup Widget. Iguana communique spécifiquement sur les mashups mis à la disposition de ses clients et l'adjonction de services afférente : superposition des horaires d'ouverture sur une carte Google, inclusion de widgets sociaux comme l'accès à Twitter, aménagement de flux RSS de n'importe quelle source. Une «communauté clients » peut 
également se constituer par la possibilité de mettre en contact les comptes d'une même famille ou d'amis. Ces derniers peuvent, ainsi, partager leur passion de la littérature ou de la musique.

\section{Encadré 2 : Toocamp, agrégateur d'informations pour un meilleur time to market}

Toocamp est un site agrégateur d'informations qui permet de rechercher et comparer des locations en camping. Les données tels que le prix, l'emplacement, la disponibilité reposent sur le référencement de plus d'un millier de sites de camping grâce à l'utilisation de la technologie mashup de Convertigo. Cette solution capte les informations sur l'ensemble des sites référencés sans partage de fichiers. Selon Toocamp, le succès du site qui a enregistré plus de 50000 visiteurs mensuels en 2010 réside dans sa capacité à intégrer en temps réel des informations extrêmement hétérogènes et changeantes. Cette agilité représente un avantage majeur sur le marché puisqu'elle rend possible les comparaisons de prix ou de services d'un nombre considérable de campings. Elle change également les usages des consommateurs qui se détachent des annuaires pour expérimenter ce type de site.

Grâce à cet aspect de catalogue étendu, interactif, homogénéisé, les mashups deviennent un outil performant de fidélisation car ils offrent aux clients une palette élargie d'informations et de services qui permet de diminuer le taux de rebond. Les consommateurs sont moins tentés de continuer leur navigation (et donc de quitter le site) pour rechercher une information manquante.

\section{- Zooming et gestion du multi-support}

Par ailleurs, l'ergonomie des catalogues est considérablement modifiée par l'utilisation des mashups. Le zooming enrichi est une technique typiquement mashup permettant de zoomer sur une image en haute résolution pour en explorer les détails tout en lui associant une ficheproduit interactive. Le mélange de l'image et de l'information-produit est développé par des sociétés comme Deep Zoom ou Zazzle qui mettent en place des interfaces regroupant texte, photos, vidéo et audio sur une même page. Cette technique assimilée à une technique de rich media (ou média enrichi) améliore l'immersion et le « ressenti virtuel » du produit, présenté sous plusieurs angles. Adepte de cette technique, le site Darty a pu constater une 
augmentation de $25 \%$ de son taux de conversion ${ }^{6}$. Enfin, l'étude Forrester de 2008 reprise par Internet Retailer (4) et portant sur l'utilisation du zooming en e-commerce montre qu'une meilleure expérience sensitive rassure le consommateur et diminue le taux de retour des articles.

\section{Préconisations managériales : du choix des API à l'évaluation des bénéfices apportés}

Ce panorama synthétique des principaux bénéfices apportés par les mashups en termes d'agilité doit s'accompagner, de la part des entreprises, d'une vraie réflexion sur leur utilisation et implémentation. La décision de recourir à un mashup ne peut être prise qu'en suivant un processus rigoureux de veille et d'évaluation des mashups existant chez ses concurrents ou dans d'autres secteurs. Les étapes sont décrites dans le schéma suivant :

Figure 1 : Etapes de sélection d'un mashup

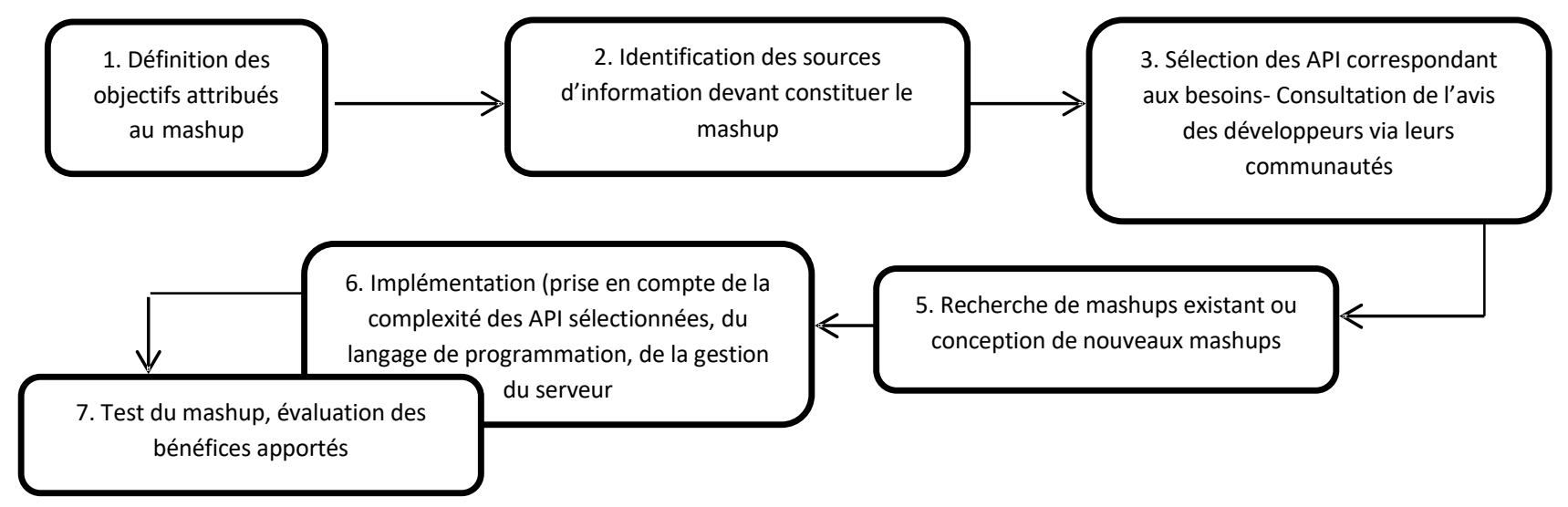

Ces différentes étapes illustrent la nécessité de disposer d'une veille active (préalable à toute démarche agile) des mashups nouveaux et des mashups les plus populaires. Très souvent développés par les consommateurs eux-mêmes, les mashups les plus utilisés sont représentatifs des besoins non encore satisfaits des consommateurs. Ainsi, des sites comme Programmable Web peuvent être considérés comme des plateformes de solutions imaginées par les utilisateurs. Ils s'inscrivent dans des logiques de crowd sourcing qui doivent être vues par les managers comme :

\footnotetext{
${ }^{6}$ Source : www.zoomorama.com. Zoomorama était une start-up innovante spécialisée dans l'ergonomie des sites Web (conseil et développement) jusqu'en 2010.
} 
- Un moyen de réduction des coûts consacrés à la recherche de nouvelles solutions agiles

- Une visualisation en temps réel des mashups en tant que catalyseurs d'innovations fondées sur les usages

Néanmoins, il convient de tenir compte des dangers de l'imitation systématique. En effet, la sélection des mashups les plus utilisés peut entraîner une perte de créativité et d'efficacité, la prime étant donnée à la popularité au détriment de la pertinence. Ainsi les phases amont d'analyse des besoins et aval de tests utilisateurs deviennent primordiales pour sélectionner les mashups les plus adaptés. Enfin, un certain nombre de questions sur la dépendance engendrée par les API demeurent. Cette dépendance serait de trois sortes :

1. Les API gratuites reposent de plus en plus sur un modèle freemium au sein duquel les fonctionnalités les plus simples sont gratuites, les plus avancées payantes.

2. Les API peuvent changer, se modifier et obligent à reconfigurer partiellement ou totalement les mashups associés.

3. Les API sont mises à disposition par les éditeurs de contenus comme Google, Facebook ou Amazon qui peuvent capter, en retour, les données de leurs utilisateurs ce qui peut poser, à terme, des problèmes de confidentialité et de protection de ces mêmes données.

\section{Conclusion, menaces sur l'agilité ?}

Si nous avons associé mashups et agilité, cette association comporte des avantages, des risques mais également une nouvelle façon de concevoir l'innovation. Les mashups représentent un réel potentiel d'agilité pour les entreprises. Ils donnent aux systèmes d'information marketing une souplesse et une ouverture sur un monde externe à l'entreprise : réseaux sociaux, données de géolocalisation, données financières. Néanmoins, cette agilité apportée par les mashups est dépendante des réactions protectionnistes de certains acteurs du marché. Ainsi, pour les experts du secteur, l'avenir des mashups passe par une accessibilité des API et une créativité des offres. L'accessibilité pourrait être remise en cause par un retrait du domaine public des API par les grands acteurs du Web : Yahoo, Google, Amazon. En effet, les API récupérées par de nouveaux entrants et intégrés à des mashups plus créatifs pourraient à terme concurrencer directement les services des fournisseurs d'API. Par ailleurs, les API français sont commercialisées via des partenaires choisis (Géoportail de l'IGN, ViaMichelin, la SNCF) et ne sont donc pas libres d'accès comme les mashups américains. Le 
développement de ce que Benavent (1) appelle la B-viralité à savoir la constitution de franchises électroniques telles qu'elles sont mises en oeuvre par Amazon en permettant le fonctionnement de boutiques spécialisées locales sur sa propre plate-forme pose la question de la notion même de marque pour les sites de e-commerce. Comment gérer la dépendance de ces sites (en terme de qualité, de contenus...) face à ces nouveaux acteurs, apporteurs de services mais également brouilleurs potentiels d'image et de e-réputation ? Ces contestations amènent certains chercheurs à reconsidérer, à la lumière du phénomène des mashups (12), les processus innovants. D'une part, les mashups font partie d'un écosystème qui donne aux innovations un caractère systémique (7). Les innovations dépendent, de plus en plus, de parties prenantes externes à l'entreprise, elles sont cumulatives voire combinatoires (8). D'autre part, la recherche de création de valeur devient immédiate tout en s'inscrivant dans une course accélérée à la production continuelle de nouveaux mashups (10). Cette agilité extrême pourrait représenter, à terme, un danger pour une agilité réfléchie, porteuse de valeur pour les marques.

\section{Références}

(1) Benavent C. (2009), Mashups ! Les données dans les nuages, une pluie de portail, Technologies du marketing, juillet.

(2) Charbonnier-Voirin A. (2009), Création et validation d'une échelle de mesure de l'agilité organisationnelle : étude exploratoire et confirmatoire, Congrès de l'AGRH 2009.

(3) Cova, V. et Cova, B. (2001), Alternatives Marketing : réponses marketing aux évolutions récentes des consommateurs, Paris, Dunod.

(4) Deatsch K. (2008), Better images boost conversions by 22\% at Fathead.com, Internet Retailer, 01-07.

(5) Erb B., Elsholz J.P. et Hauck F.J. (2009), Semantic Mashup, Mashing up information in the Todays World Wide Web, an overview, White Paper, VS-R08-2009, Institute of distributed systems, ULM University.

(6) Joroff M. (2002), Redefining the workplace with agility, IIE Solutions, 34, 7.

(7) Maula M., Keil T. et Salmenkaita J-P. (2006), Open Innovation in system innovation contexts, 12, in : Chesbrough H., Vanhaverbeke W., West J. (eds), Open Innovation : Researching a new paradigm, Oxford, Oxford University Press, 249-257. 
(8) Navarre C. (2005), Le futur du e-commerce : la Chine, les systèmes d'enchère au service de l'écologie et la gouvernance « chaordique », in Badot, O. et Benoun, M. (ed), Commerce et Distribution : Prospective et Stratégies, Economica, Paris, 183-191.

(9) Roberts N. et Grover V. (2012), Leveraging Information Technology Infrastructure to Facilitate a Firm's Customer Agility and Competitive Activity: An Empirical Investigation, Journal of Management Information Systems, 28, 4, 231-269.

(10) Sawhney M., Verona G. et Prandelli E. (2005), The Internet as a platform for customer engagement in product innovation, Journal of Interactive Marketing, 19,4,417.

(11) Van Oosterhout M., Waarts E. et van Hillegersberg J. (2006), Change factors requiring agility and implications for IT, European Journal of Information Systems, $15,2,132-145$.

(12) Weiss M. et Gandharan G.R. (2010), Modeling the mashup ecosystem: structure and growth, $R \& D$ Management, 1, 40, 40-49.

(13) Young O. (2008), The mashup opportunity, how to make money in the evolving mashup ecosystem, Forrester Research Inc. 